\title{
Deutsche und albanische Phraseologismen mit Tierbezeichnungen im Kontrast
}

\author{
Milote Sadiku (Prishtina)
}

\begin{abstract}
The object of this article are animal idioms in contemporary German and Albanian: the article treats those units that denote animal names in their formal structure with phraseological, figurative meaning. The aim of the article is to identify and describe the differences and similarities between these two languages from the viewpoint of a contrastive analysis. The German phraseology has often been the subject of inter-linguistic comparisons. However, this can not be claimed for the German - Albanian language analysis. In this article, some similarities and differences in animal symbolism in the German and Albanian phraseology are illustrated by typical examples, and equivalence relations between the German and Albanian animal idioms are identified.

It can be concluded that the animal names in idiom units indicate positive and negative characteristics or behavior of people. One result is that the negative semantic characteristics predominate in both languages. The animal name depends on a concrete idiom not only from the ideas and values that are attributed to an animal in a language community, but also from the already existing structures and pragmatic rules in a concrete language.
\end{abstract}

\section{$1 \quad$ Einleitung}

Das Untersuchungsobjekt des vorliegenden Beitrages sind Phraseologismen mit Tierbezeichnungen im gegenwärtigen Deutschen und Albanischen, d.h. Benennungseinheiten mit phraseologisch gebundener, figurativer Bedeutung, die in ihrer formalen Struktur einen Tiernamen enthalten ${ }^{1}$.

Tierbezeichnungen bilden in beiden Sprachen eine der größten und produktivsten Konstituentengruppen in Phraseologismen (cf. Dobrovols'kij/Piirainen 1997: 158, Fleischer 1997: 184). Der Beitrag setzt sich das Ziel, die Divergenzen bzw. Übereinstimmungen zwischen beiden Sprachen in einer kontrastiven Analyse festzustellen und zu beschreiben. Die untersuchten Phraseologismen wurden folgenden Wörterbüchern entnommen:

1. Für das Deutsche:

- Band 11 der Duden-Reihe Redewendungen. Wörterbuch der deutschen Idiomatik (1998) und

- H. Schemann: Deutsche Idiomatik. Die deutschen Redewendungen im Kontext (1993).

2. Für das Albanische:

\footnotetext{
1 Diese Benennungen bilden auch den Hauptgegenstand meines Promotionsvorhabens, das sich unter sprachvergleichendem Aspekt mit den Besonderheiten von ausgewählten Phrasemen, d. h. von Phrasemen mit Tierbezeichnungen, in der Vermittlung von Deutsch als Fremdsprache für albanische Muttersprachler befasst.
} 
- J. Thomaj (1999): Fjalor frazeologjik i gjuhës shqipe (,Phraseologisches Wörterbuch der albanischen Sprache') und

- A. Kostallari et al. (2002): Fjalor i gjuhës së sotme shqipe ('Wörterbuch der albanischen Sprache der Gegenwart').

3. Zur Durchführung der kontrastiven Analyse wurde zusätzlich das Wörterbuch Deutschserbokroatisches phraseologisches Wörterbuch von Mrazović/Primorac zu Rate gezogen (Mrazović/Primorac 1991).

In die Zielstellung der kontrastiven Analyse gehen folgende Fragen ein:

1. Welche Unterschiede bestehen in der deutsch-albanischen Tiersymbolik?

2. Welche Äquivalenzbeziehungen bestehen zwischen deutschen und albanischen Phraseologismen mit Tierbezeichnungen?

3. Haben kulturell bedingte Unterschiede in der Tiersymbolik einen Einfluss auf Äquivalenzbeziehungen in der Phraseologie der beiden Sprachen? Wie können diese durch die Analyse von deutschen und albanischen Phraseologismen festgestellt werden?

Insgesamt wurden 283 deutsche Phraseologismen, die den genannten deutschen Wörterbüchern entstammen, untersucht. Sie zählen zu den gebräuchlichen Phraseologismen der deutschen Gegenwartssprache. Es handelt sich bei ihnen um einen Kernbereich eindeutig zu identifizierender fester Wendungen (cf. DUDEN 11 1998: 13). Ihre Auswahl wurde nicht nur auf der Grundlage von allgemeinen und speziellen Wörterbüchern vorgenommen, sondern auch auf der breiten Materialbelegsammlung der Dudenredaktion und von Schemanns (1993) deutscher Idiomatik. ${ }^{2}$ Ihnen werden vergleichend 235 albanische Phraseologismen gegenübergestellt. Es sind die Äquivalente, die den deutschen Beispielen auf der Grundlage der aufgeführten albanischen Wörterbücher entsprechen. Weitere Aussagen zu albanischen Phraseologismen sind somit kommenden Analysen vorbehalten.

Die deutsche „Phraseologie ist oft Gegenstand interlingualer Vergleiche gewesen mit unterschiedlicher Zielsetzung.“ (Dobrovol'skij/Piirainen 1997: 47), d.h. Teile des deutschen phraseologischen Systems wurden mehrfach mit respektiven Teilsystemen anderer Sprachen kontrastiert. Dies lässt sich allerdings für das Sprachenpaar Deutsch - Albanisch nicht behaupten, denn die einzige größere kontrastive Arbeit manifestiert sich in einem phraseologischen Wörterbuch Deutsch - Albanisch von Skender Doku (Doku 1998). Somit besitzt die kontrastive Analyse des Sprachenpaars Deutsch - Albanisch nur eine marginale Stellung in der kontrastiven Phraseologie. Verglichen mit der großen Fülle von Veröffentlichungen der deutschsprachigen Phraseologie ist die Auseinandersetzung mit phraseologischen Fragestellungen in der Albanistik ebenfalls nur bruchstückhaft vorhanden.

Eine allgemeine Definition des zu untersuchenden Sprachmaterials bezieht sich auf Fleischer (1997: 29), Burger (2010: 14) und Wotjak (1992: 3). Demnach liegt dieser Arbeit eine Klassifikation nach den Eigenschaften der Mehrgliedrigkeit, der relativen Festigkeit, Reproduzierbarkeit und Idiomatizität zugrunde. Phraseologismen werden als (relativ) stabile sprachliche Einheiten verstanden, die die Eigenschaften Polylexikalität, Festigkeit, (Phraseologismen im weiteren Sinn) und Idiomatizität (Phraseologismen im engeren Sinn) aufweisen, die durch Lexikalisierung und Reproduzierbarkeit gekennzeichnet sind. Mit der Idiomatizität wird die Diskrepanz zwischen der phraseologischen Bedeutung und der wörtlichen Bedeutung bezeichnet (cf. Burger 2010: 30). Der Satz gilt als die obere formale Grenze phraseologischer Einheiten (cf. Burger 2010: 15). In die Analyse ist der durchaus weit gefassten Begriffsdefinition zufolge ein relativ umfangreiches und vielfältiges Sprachmaterial aufzunehmen: alle Phraseologismen, in denen Tierbezeichnungen vorkommen.

\footnotetext{
2 Cf. DUDEN 11 1998: 847, Schemann 1993: XI.
} 
Földes betrachtet Phraseologismen als einen prototypischen Hort des „kulturellen Gedächtnisses" einer Sprachgemeinschaft, in dem sich das versprachlichte kollektive Wissen und damit das ,sprachliche Weltbild“ in aufschlussreicher Weise manifestiert (Földes 2007: 432). Da deutsche und albanische Sprecher eine unterschiedliche Geschichte, Natur, Kultur und daraus resultierende Unterschiede in der Wahrnehmung der Welt haben, ist für die vorliegende Untersuchung davon auszugehen, dass sich in Phraseologismen kulturspezifische und kulturtypische Begebenheiten widerspiegeln.

Bevor ich im Folgenden zu der zwischensprachlichen Äquivalenz in der kontrastiven Phraseologie übergehe, werde ich anhand einiger typischer Beispiele einige Gemeinsamkeiten und Unterschiede in der Tiersymbolik in der deutschen und der albanischen Phraseologie darstellen. Sie helfen, da das Albanische im europäischen und anderen Sprachräumen nur begrenzt vermittelt und gesprochen wird, die deutsch-albanische Äquivalenzproblematik besser zu verstehen.

\section{Tiersymbolik in Phraseologie}

In vielen Kulturen haben Tiere eine symbolische Funktion. In allen Kulturgemeinschaften wurden den

[...] Tieren bestimmte symbolische Funktionen zugeschrieben, wurden sie in ihrem Verhalten, ihrer äußeren Erscheinung, ihrer Beziehung zum Menschen usw. mit symbolischen Bedeutungen belegt. (Dobrovol'skij/Piirainen 1997: 157)

Es ist vom entsprechenden Kulturraum abhängig, welche Eigenschaften mit dem jeweiligen Tier assoziiert werden. Dobrovol'skij/Piirainen heben hervor, dass Tiermetaphorik, d.h. Übertragungen aus dem Tierreich auf Verhaltens- oder Handlungsweisen des Menschen, in Parömien und Idiomen eine wichtige Rolle spielen, sowie dass die Tiersymbolik oft deutlicher in Sprichwörtern als in Idiomen hervortritt. In der deutschen Sprache gibt es hunderte von Phraseologismen, die auf Tiere jeder Art (auf wilde Tiere, Haustiere, Kleintiere usw.) bezogen sind (cf. Dobrovol'skij/Piirainen 1997: 159). Durch die Untersuchung dieser speziellen Gruppe von Phraseologismen lassen sich Übereinstimmungen und Divergenzen in den Symbolfeldern und Kulturen erkennen, z.B. welche auf den Menschen übertragbare Eigenschaften den Tieren zugeschrieben werden. So stellt sich die Frage, warum man im Deutschen hungrig wie ein Bär/Hund/Wolf ist, während man im Albanischen nur wie ein ,Wolf' hungrig sein kann ,i uritur si ujk' (cf. Burger et al. 1982: 36).

Aus dem Vergleich der deutschen und albanischen Phraseologismen mit Tierbezeichnungen konnte festgestellt werden, dass die Tierkomponente in Phraseologismen überwiegend negative Eigenschaften oder Verhaltensweisen des Menschen bezeichnen. Die folgenden Beispiele mit den Komponenten Hund ,qen', Hase ,lepur', Bär ,ari', Wolf , ujk' und Schlange ,gjarpër' zeigen diese Tendenz deutlich:

1. HUND: „Im kulturellen Bereich ist 'Treue' die alle anderen überragende symbolische Bedeutung des Hundes“ (Dobrovol'skij/Piirainen 1997: 207). Sprache und Kultur fallen jedoch nicht völlig zusammen, denn es konnten keine deutsche Phraseologismen gefunden werden, in denen Hund in der symbolischen Funktion des treuen Charakters im positiven Sinne begegnet. Im Albanischen gibt es zwar die Einheit qen besnik 'treuer Hund', doch ,Treue' ist in diesem Beispiel im Sinne von ,Unterwürfigkeit' eher negativ konnotiert, also ,ein gehorsamer und einem anderen unterworfener Mensch'. Bei den Phraseologismen mit einer Bezeichnung des Hundes als Komponente erkennt man die Bedeutung der

- 'Minderwertigkeit':

dt. leben wie ein Hund, alb. jeton si qen 'in erbärmlichen Umständen leben' 
- 'Boshaftigkeit, Aggressivität':

dt. keine schlafenden Hunde wecken 'nicht unnötig jmds. Aufmerksamkeit erregen und sich damit überflüssige Unannehmlichkeiten bereiten' (cf. Dobrovol'skij/Piirainen 1997: 202)

alb. qen bir qeni (dt. *Hundesohn) 'ein sehr böser und gemeiner Mensch'

- 'Streitsucht':

dt. leben wie Hund und Katze

alb. shkojnë si qeni me macen (wörtl. *sie vertragen sich wie Hund und Katze) 'sich ständig streiten'

2. HASE: Mit der Komponente Hase wird in beiden Sprachen die Vorstellung von 'Ängstlichkeit' und 'Feigheit' verbunden:

- 'sehr ängstlich sein':

dt. ängstlich wie ein Hase

alb. $u$ bë lepur (wörtl. *jmd. wurde zum Hasen)

- 'viel Angst bekommen und weglaufen':

alb. ka lepurin në bark (wörtl. *jmd. hat den Hasen im Bauch) 'jmd. ist ein großer Feigling'.

Im Deutschen tritt Hase noch mit der Bedeutung 'Erfahrenheit' in ein alter Hase sein auf. Im Albanischen wird der Hase nicht mit dieser Bedeutung verbunden; im Zusammenhang mit 'Erfahrenheit' wird aber statt der Komponente Lepur 'Hase' die Komponente Ujk 'Wolf' verwendet: është ujk $i$ vjetër (*ein alter Wolf sein) 'ein erfahrener Mensch'3.

3. BÄR: Der Bär wird im Deutschen und Albanischen mit der Vorstellung von 'Stärke' assoziiert und negativ konnotiert im Sinne von 'Nachteil, Schaden'. Der interlinguale Unterschied liegt in der Genuswahl der Komponente Bär, denn in den albanischen Phraseologismen wird nicht die männliche Form ari-u, sondern immer die weibliche Form arushë verwendet:

- ,Stärke“:

dt. stark wie ein Bär

alb. si arushë (wörtl. *wie eine Bärin)

alb. arushë mali (wörtl. *Bärin des Waldes) 'sehr starke Person'

- 'Nachteil, Schaden':

dt. jmdm. einen Bärendienst erweisen 'jmdm. einen schlechten Dienst erweisen' alb. ia la këmbët e arushës në dorë (wörtl. *jmdm. Bärenbeine (Bärenpfoten) in der Hand lassen) 'jmds. Untreue wird ironisiert'

Die Bedeutung 'Hunger, Gier' ist nur im Deutschen mit dem Bär verbunden (dt. hungrig wie ein Bär sein). Im Albanischen wird 'Hunger' nur durch die Komponente ujk 'Wolf' in $i$ uritur si ujk (*hungrig wie ein Wolf) in der Bedeutung 'sehr hungrig' versprachlicht.

4. WOLF: Die Komponente Wolf wird in beiden Sprachen in Verbindung mit ,Hunger', ,Gefahr', ,Boshaftigkeit und Aggressivität' verwendet:

\footnotetext{
${ }^{3}$ Im Albanischen existiert noch die Einheit është dhelpër plakële vjetër (wörtl. *eine alte Füchsin sein), die sowohl mit der Bedeutung ,ein erfahrener Mensch' als auch mit ,ein sehr listiger Mensch' verbunden wird.
} 
Milote Sadiku: Deutsche und albanische Phraseologismen mit Tierbezeichnungen im Kontrast 145

- 'Hunger':

dt. hungrig wie ein Wolf

alb. i uritur si ujk

- 'Gefahr':

dt. ein Wolf im Schafsfell

alb. ujk me lëkurë qengji (wörtl. *ein Wolf im Lammfell)

- 'Boshaftigkeit, Aggressivität':

dt. mit den Wölfen heulen' sich der Mehrheit (aus Opportunismus) anschließen, etwas moralisch nicht Einwandfreies gegen die eigene Überzeugung mitmachen'

alb. ujku qimen e ndërron, zanatin se harron (wörtl. *der Wolf ändert das Haar, vergießt die Gewohnheit nicht) 'schlechter Charakter und aggressives Verhalten lässt sich niemals ablegen oder ändern'.

5. SCHLANGE / NATTER: Mit der Schlange/Natter lässt sich das Symbol der 'Falschheit, Boshaftigkeit' in beiden Sprachen verbinden:

dt. eine Schlange/Natter am Busen nähren

alb. rris/mbaj gjarpërin në gji (wörtl. *die Schlange am Busen großziehen/halten) 'jmdn. in seine Obhut nehmen, von dem man später geschädigt wird oder Schaden zu befürchten hat'.

alb. është nepërkë (wörtl. eine Natter sein), ein sehr schlechter und untreuer Mensch'

Im Albanischen verbindet man mit der Schlange das Symbol der 'Gefahr':

alb. gjarpër me zile / me dy koka (wörtl. *eine Schlange mit Glocke/ mit zwei Köpfen) 'ein sehr gefährlicher Mensch'.

Die Beispiele zeigen im analysierten phraselogischen Teilbereich zumeist Korrespondenzen zwischen dem Deutschen und Albanischen. Die Unterschiede konzentrieren sich auf eine unterschiedliche Gewichtung von Konnotationen (s. 1), auf fehlenden Konnotationen in einer der beiden Sprachen (s. 2, 3, 5) sowie auf einen unterschiedlichen denotativen Genusbezug von Tierbezeichnungen (s. 3). ${ }^{4}$

\section{Zwischensprachliche Äquivalenz in der deutsch-albanischen Phraseologie}

Bei einem interlingualen Vergleich phraseologischer Einheiten kann Korhonen (2007: 575) zufolge entweder die denotative Bedeutung (= semantische oder inhaltliche Äquivalenz) oder die Form (= formale oder morphosyntaktisch-lexikalische Äquivalenz) als Ausgangspunkt dienen. In den meisten Fällen wird bei der Suche nach äquivalenten phraseologischen Einheiten jedoch von der denotativen Bedeutung ausgegangen, die ,als unerlässliche Voraussetzung für interlinguale Kontrastierung von Phraseologismen mit praktischer Zielsetzung“ (Korhonen 2007: 575) gilt. Korhonen weist darauf hin, dass bei der Kontrastierung der denotativen Bedeutung gewisse Asymmetrien auftreten können, etwa dadurch, dass die Zahl der denotativen Bedeutung unterschiedlich ist, d.h. ein Phraseologismus des Deutschen kann polysem, sein Äquivalent des Albanischen hingegen monosem sein oder umgekehrt: So lässt sich dem deutschen wie eine gesengte Sau das albanische (iki) si derr/gjel i plagosur (wörtl. *(lief davon) wie ein verwundetes Schwein/ verwundeter Hahn) als Äquivalent zuweisen, allerdings nur mit der denotativen Bedeutung

\footnotetext{
${ }^{4}$ Weiteres ist durch künftige Untersuchungen aufzuzeigen.
} 
,sehr schnell (in Bezug auf das Weglaufen vor Angst o.ä)' und nicht mit der denotativen Bedeutung ,fruchtbar schlecht (in Bezug auf das Verhalten)'.

Die denotative Bedeutung der verglichenen Einheiten wird als dominierender äquivalenzbestimmender Faktor angesetzt. Gemäß der Eigenheit und Komplexität des Untersuchungsobjektes dürfen jedoch die zugrunde gelegten Äquivalenzkriterien nach Földes (1996: 117) „nicht lediglich auf eine Ebene der Sprachbeschreibung beschränkt bleiben“. Zu weiteren Äquivalenzparametern zählen u.a. Struktur ${ }^{5}$, Idiomatizität, Bildhaftigkeit, Stabilität und Pragmatik (Konnotation). Korhonen (2007) zählt als weiteren Faktor ebenso die Valenz.

In der kontrastiven Phraseologie wird Äquivalenz in quantitative und qualitative Äquivalenz unterteilt (cf. Földes 1996: 117; Korhonen 2007: 577). Die Ermittlung der Äquivalenztypen basiert hier auf der qualitativen Äquivalenz zwischen den verglichenen Einheiten. In der kontrastiven Phraseologie besteht in diesem Zusammenhang eine weit gehende Einigkeit über die dabei zu unterscheidenden Grundtypen phraseologischer Äquivalenz. Allerdings variiert in Abhängigkeit von der Berücksichtigung der unterschiedlichen Äquivalenzparameter (cf. dazu Korhonen 2007) die konkrete Zuweisung einzelner Phrasempaare zu diesen Grundtypen. Den nachstehenden Analyseergebnissen liegt die Klassifikation Korhonens (2007) zugrunde. In dieser Klassifikation haben sich die Kategorien Volläquivalenz, Teiläquivalenz und Ersatzäquivalenz als angemessen für die Beschreibung von interlingualen Ähnlichkeiten und Unterschieden erwiesen.

\subsection{Volläquivalenz}

Die Volläquivalenz bzw. totale Äquivalenz kann Korhonen zufolge als Idealfall von Äquivalenz angesehen werden (cf. Korhonen 2007: 578). Volläquivalente Phraseologismen müssen in allen wesentlichen Äquivalenzparametern miteinander übereinstimmen, d.h. zwischen den kontrastierten Einheiten muss die gleiche denotative und konnotative, emotional-expressive und stilistische Gesamtbedeutung sowie die völlige Kongruenz in der Komponentenkette liegen (cf. auch Földes 1996: 118). Die Übereinstimmung zweier Einheiten in allen Vergleichsparametern ist in der deutschen und der albanischen Sprache nicht sehr häufig. Korhonen (2007: 578) vertritt bezogen auf volläquivalente Fälle auch die Meinung, dass die meisten Phraseologismen dieses Äquivalenztyps phraseologische Internationalismen sind, „die auf einem ähnlichen kulturellen Hintergrund fußen“. Er weist darauf hin, dass die Phraseologismen oft aus der Bibel, aus der antiken Literatur und Mythologie stammen oder dass einige phraseologische Äquivalente Entlehnungen sein können (cf. Korhonen 2007: 578).

Im untersuchten Material konnten 33 phraseologische Paare ermittelt werden, die diesen Annahmen entsprechen und sich als volläquivalent bezeichnen lassen, z.B.:

- dt. weder Fisch noch Fleisch - alb. as mish as peshk

- dt. sterben wie die Fliegen - alb. vdiqën si mizat

- $\quad$ dt. wenn der Hahn Eier legt - alb. kur të bëjë gjeli vezë

- dt. mit den Hühnern aufstehen - alb. zgjohem me pula

\footnotetext{
${ }^{5}$ Für die Struktur wäre darauf hinzuweisen, dass bei der Gegenüberstellung der deutschen und der albanischen Sprache von einem bestimmten morphosyntaktischen Unterschied abgesehen werden muss, so z.B. vom NichtVorkommen des unbestimmten Artikels im Albanischen. Bei den albanischen Einheiten ist im Allgemeinen zu beobachten, dass der unbestimmte Artikel nicht verwendet wird, da die Unbestimmtheit an der Form des Substantivs klar wird. Außerdem erscheint es wichtig zu erwähnen, dass der bestimmte Artikel im Albanischen dem Substantiv nachgestellt wird und dass er mit ihm als eine Einheit fungiert, z. B. qen ,Hund' - qeni ,der Hund' (-i ist der männliche bestimmte Artikel).
} 
- dt. leben wie ein Hund - alb. jeton si qen

- dt. hungrig wie ein Wolf - alb. $i$ uritur si ujk

- dt. arbeiten wie ein Pferd - alb. punon si kalë

- dt. (sich fühlen) wie ein Fisch im Wasser - alb. (ndihet) si peshku në ujë

- dt. wie ein geprügelter Hund - alb. si qen i rrahur

Der durchgeführte deutsch-albanische Sprachvergleich zeigt, dass in einem ausgewählten Teilbereich von Phraseologismen mit Tierbezeichnungen nur $12 \%$ von Phrasemen mit Volläquivalenz vorhanden sind. Allerdings nehmen sie innerhalb einzelner Äquivalenzgruppen, wie die nachstehenden Ausführungen zeigen, die erste Stelle ein.

\subsection{Teiläquivalenz}

Eine Teiläquivalenz bzw. partielle oder approximative Äquivalenz liegt dann vor, wenn es Abweichungen in einem oder in mehreren Äquivalenzparametern gibt, wobei die (relative) Gleichheit in der denotativen Bedeutung ausschlaggebend für die Zuordnung der phraseologischen Paare zur Teiläquivalenz ist. Die Unterschiede zwischen den kontrastierten Einheiten können z.B. im Bereich der Struktur (morphosyntaktische und/oder lexikalische Unterschiede im Komponentenbestand der Phraseme), in der Stilebene (Konnotationen etc.) oder in der semantischen und syntaktischen Verknüpfbarkeit bestehen.

Bei der kontrastiven Untersuchung der deutschen und albanischen phraseologischen Einheiten mit Tiernamen konnten folgende Untertypen von Teiläquivalenz unterschieden werden:

a) Eine stark vertretene Gruppe innerhalb der Teiläquivalenz besteht aus Phraseologismen, die semantische und strukturelle Ähnlichkeiten aufweisen. Sie haben die gleiche denotative Bedeutung; es lassen sich aber Unterschiede in der Lexem- bzw. Komponentenstruktur und somit der wörtlichen Bedeutung feststellen. Im untersuchten Material konnten 23 phraseologische Paare $(=8 \%)$ dieser Gruppe zugeordnet werden, z.B.:

- dt. ein Wolf im Schafspelz - alb. ujk me lëkurë qengji (wörtl. *ein Wolf im Lammpelz)

- dt. ein alter Hase sein - alb. [është] ujk $i$ vjetër (wörtl. *[ist] ein alter Wolf)

- dt. ein weißer Rabe - alb. një mizë e bardhë (wörtl. *eine weiße Fliege)

- dt. zwei Fliegen mit einer Klappe schlagen -alb. me një gur vras dy zogj (wörtl. *mit einem Stein zwei Vögel töten)

- dt. die Katze im Sack kaufen - alb. blej derr në thes (wörtl. *ein Schwein im Sack kaufen)

- dt. aus einer Mücke einen Elefanten machen - alb. mizën e bën buall (wörtl. *die Fliege zum Bullen machen)

- dt. einem geschenkten Gaul schaut man nichts ins Maul - alb. kalit të falur s'i shikohen dhëmbët (wörtl. *dem geschenkten Pferd schaut man nicht die Zähne)

- dt. Hahn im Korb[e] sein - alb. (jam) si gjel midis pulash (wörtl. *[sein] wie Hahn unter Hühnern)

- dt. die Schafe von den Böcken trennen/scheiden - alb. ndaj delet nga dhitë (wörtl. *die Schafe von den Ziegen trennen)

b) Eine zweite Gruppe bilden Phraseologismen mit gleicher denotativer Bedeutung aber völlig unterschiedlicher Komponentenstruktur und somit einer anderen wörtlichen Bedeutung. Die Phraseologismen entstammen somit verschiedenen Bildspenderbereichen. Dies ist mit 
insgesamt 67 phraseologischen Paaren $(=24 \%)$ die größte Gruppe innerhalb der teiläquivalenten Phraseologismen:

- dt. wenn der Hund mit dem Schwanz bellt - alb. kur të hip gomari në fik (wörtl. *wenn der Esels auf den Feigenbaum steigt)

- dt. beim Esel Wolle suchen - alb. nxjerr dhjamë nga pleshti (wörtl. * Speck vom Floh erhalten wollen)

- dt. aus jmdm. einen Affen machen - alb. luaj me të si me arushë (wörtl. *spielen mit ihm wie mit [einer] Bärin)

- dt. etw. verstehen wie die Kuh vom Radfahren /Sonntag - alb. s'di si (nga) lidhet gomari (wörtl. *[jmd.] weiß nicht wie wird der Esel gebunden) / s'di t’i japë ujë (as) gomarit (wörtl. *[jmd.] weiß nicht mal dem Esel Wasser zu geben)

- dt.für die Katz sein - alb. për dhjamë qeni (wörtl. *für Speck des Hundes)

- dt. der Esel geht voran - alb. del si mëzi para pelës (wörtl. *gehen wie das Fohlen vor der Stute)

c) Eine weitere Gruppe besteht aus phraseologischen Einheiten, die in der denotativen Bedeutung und in der Stilschicht sowie der wörtlichen Bedeutung übereinstimmen, jedoch morphosyntaktische Unterschiede bei den Komponenten aufweisen. Hierbei entstammen beide Phraseologismen dem gleichen Bildspenderbereich. Im untersuchten Material konnten 25 phraseologische Paare $(=9 \%)$ ermittelt werden, die sich diesem Untertyp der Teiläquivalenz zuordnen lassen:

- dt. Hunde, die bellen, beißen nicht - alb. qeni qi leh s't'han (wörtl. *der Hund, der bellt, beißt nicht). Die deutsche Wendung ist im Plural und die albanische im Singular gebräuchlich.

- dt. (stark) wie ein Bär - alb. si arushë (wörtl. *wie eine Bärin). In der albanischen Wendung wird die Komponente mit der Tierbezeichnung in der weiblichen Form gebraucht im Gegensatz zur männlichen Form der deutschen Wendung.

- dt. die Hunde bellen, und/aber die Karawane zieht weiter - alb. qentë le të lehin, Karavani shkon përpara (wörtl. *Lass die Hunde bellen, die Karawane geht voran). Die deutsche Wendung, die Hunde bellen' ist im Indikativ und die albanische ,qentë le të lehin' im Jussiv 6 gebräuchlich.

- dt. glatt wie ein Aal sein - alb. rrëshqet si ngjalë (wörtl. * wie Aal gleiten)

- dt. der Berg kreißte und gebar eine Maus - alb. u mbars mali, polli mi (wörtl. *der Berg wurde schwanger, gebar eine Maus)

- dt. leben wie Hund und Katze - alb. jetojnë si qeni me macen (wörtl. *leben wie der Hund mit der Katze) ${ }^{7}$

- dt. mit jmdm. Katz und Maus spielen - alb. luan me të si macja me miun (wörtl. * mit ihm/ihr wie die Katze $\underline{\text { mit }}$ der Maus spielen)

d) Eine kleine Gruppe bilden Phraseologismen mit gleicher Form aber mit Unterschieden in der Bedeutungsstruktur. Im untersuchten Material gehören insgesamt vier phraseologische Paare $(=1 \%)$ zu dieser Gruppe. Eine Asymmetrie in der Semementsprechung besteht bei:

${ }^{6}$ Die Form des Jussivs im Albanischen drückt vor allem Gleichgültigkeit des Sprechers gegenüber der Ausführung oder dem Verlauf eines Ereignisses aus.

${ }^{7}$ Im Albanischen existiert noch die Variante shkojnë si macja me miun (wörtl. *sich vertragen wie die Katze mit der Maus). 
- dt. auf dem hohen Roß sitzen / sich aufs hohe Roß setzen und alb. është hipur në majë të kalit (wörtl. *[man] ist hoch aufs Pferd gestiegen).

Die Bedeutung der deutschen Wendungen wird im DUDEN: Deutsches Universalwörterbuch (DUW) angegeben mit ,hochmütig, überheblich sein', eine hochmütige, überhebliche Haltung annehmen', während die Bedeutung der albanischen Wendung im FGJSSH ${ }^{8}$ mit ,bei jmdm. ist alles in Ordnung, läuft alles gut' bezeichnet wird.

- dt. vom hohen Roß heruntersteigen und alb. ra (zbriti) nga kali (wörtl. *herunterfallen [heruntersteigen] vom Pferd').

Die albanische phraseologische Einheit wird im FGJSSH mit zwei Bedeutungen angegeben: 1. ,(jmd.) blieb in der Mitte des Weges, den er begonnen hatte' und 2. ironisch, ,(jmd.) fiel aus seiner hohen Position herunter, (jmd.) wurde aus seinem hohen Dienst gefeuert, (jmd) ist weder reich noch mächtig mehr', während die deutsche Wendung laut DUW die Bedeutung , seine Überheblichkeit ablegen' hat.

- dt. jmdm. auf die Hühnerauge treten und alb. e shkeli në kallo (dikë) (wörtl. *[jmdm] auf den Hühnerauge treten').

Für den deutschen Phraseologismus werden im DUW die Bedeutungen 1.,jmdn. mit einer Äußerung, einem bestimmten Verhalten an einer empfindlichen Stelle treffen' und 2. ,jmdn. nachdrücklich an etwas erinnern, was er noch nicht erledigt hat' angegeben, während für den albanischen Phraseologismus im FGJSSH nur die Bedeutung, er berührte ihn da, wo es ihm mehr weh tut, in der Wunde' angegeben wird.

e) Eine ebenfalls kleine Gruppe (1\% des gesamten Materials) bilden Phraseologismen, bei denen eine Ähnlichkeit in der wörtlichen Bedeutung vorliegt, wobei sie morphosyntaktische Unterschiede sowie eine Asymmetrie in der Semementsprechung aufweisen. Zu dieser Gruppe gehören z.B. der deutsche Phraseologismus es ist eine Maus im Mehl und der albanische Phraseologismus (është) si miu në miell (wörtl. *[sein] wie eine Maus im Mehl). Die Bedeutung des deutschen Phraseologismus lautet ,es besteht irgendein Hindernis/ Fehler; diese Sache ist nicht in Ordnung' (cf. Mrazovic/Primorac 1991: 577), während der albanische Phraseologismus im FGJSSH mit der Bedeutung, jmd. befindet sich mitten allem, was er braucht' versehen wird. Das andere kontrastierte Paar dt. der Katze die Schelle umhängen und alb. $i$ vë zile miut (wörtl. *der Maus die Schelle umhängen) weist eine ähnliche Struktur auf, aber sie entstammen unterschiedlichen Bildspenderbereichen.

Ein weiterer Unterschied liegt bei diesen Beispielen in der Semementsprechung. Die Bedeutung der deutschen Wendung wird im DUW mit, gefährliche, schwierige Aufgabe als einziger übernehmen' angegeben, während die Bedeutung der albanischen Wendung im FGJSSH mit ,jmd. erdenkt Unwarheiten, verleumdet und verbreitet nie passierende Sachen' angegeben wird.

Ähnlichkeiten in der wörtlichen Bedeutung führen, wie die Beispiele zeigen, zu unterschiedlichen Bedeutungen von Phrasemen des Deutschen und des Albanischen, nicht aber gleichfalls zu weiteren Ähnlichkeiten.

\subsection{Ersatzäquivalenz (Nulläquivalenz)}

Ersatz- oder Nulläquivalenz bedeutet, dass einem Phraseologismus der Ausgangssprache kein Phraseologismus in der Zielsprache entspricht. Die Bedeutung des ausgangssprachlichen Phraseologismus wird in der Zielsprache ,mit Hilfe von nichtphraseologischen Entsprechungen wie freien syntaktischen Wortverbindungen, Wortbildungskonstruktionen

8 Wörterbuch der albanischen Gegenwartssprache (2002). 
und primären Einzellexemen ausgedrückt" (Korhonen 2007: 581). Die denotative Bedeutung der deutschen Phraseologismen kann im Albanischen durch Paraphrasierung ausgedrückt werden. Dabei geht aber eine mögliche pragmatische Wirkung meist verloren (cf. Földes 1996: 126).

Im Korpus konnten 128 Paare $(=45 \%)$ ermittelt werden, die sich der Gruppe mit Nulläquivalenz zuordnen lassen, z.B.:

- dt. sich wie ein Elefant im Porzellanladen benehmen - wörtl. alb. nga pakujdesia e madhe dëmtoj shumë gjëra (, aus großer Ungeschicklichkeit viel Schaden errichten')

- dt. jmdm. den roten Hahn aufs Dach setzen - wörtl. alb. $i$ djeg shtëpinë dikujt (,jmdm das Haus verbrennen')

- dt. das Ei unterm Huhn verkaufen müssen - wörtl. alb. dikush ka nevojë urgjente për para (,jmd hat dringendes Bedürfnis nach Geld‘)

- dt. nicht ganz hasenrein wörtl. alb. dikush është i dyshimtë (,jmd ist verdächtig')

- dt. frieren wie ein junger Hund - wörtl. alb. kam shumë të ftohtë (wörtl. ,es ist mir sehr kalt').

\section{$4 \quad$ Zusammenfassung und Ausblick}

Zusammenfassend kann mit der vorgelegten Untersuchung festgehalten werden, dass die Tierbezeichnungen in phraseologischen Einheiten positive und negative Eigenschaften oder Verhaltensweisen des Menschen bezeichnen, wobei festzustellen ist, dass die negativen semantischen Eigenschaften in beiden Sprachen überwiegen. Hierzu kommen in beiden Sprachen gleiche, aber auch unterschiedliche Tierbezeichnungen und eine mit ihnen verbundene konvergierende bzw. divergierende Tiersymbolik zur Anwendung. Für negative Eigenschaften stehen im Deutschen und im Albanischen, wie das bisherige Belegmaterial erkennen lässt, vor allem: alb. qen besnik (wörtl. treuer Hund) ,ein gehorsamer und einem anderen unterworfener Mensch', dt. ein Wolf im Schafsfell, alb. ujku qimen e ndërron, zanatin se harron (wörtl. *der Wolf ändert das Haar, vergisst die Gewohnheit nicht) 'schlechter Charakter und aggressives Verhalten lässt sich niemals ablegen oder ändern', alb. gjarpër me zile / me dy koka (wörtl. *eine Schlange mit Glocke/ mit zwei Köpfen) 'ein sehr gefährlicher Mensch', alb. është nepërkë (wörtl. eine Natter sein), ein sehr schlechter und untreuer Mensch'. Und umgekehrt stehen in beiden Sprachen z.B. dt. ein alter Hase sein alb. është ujk $i$ vjetër (*ein alter Wolf sein) 'ein erfahrener Mensch', dt. stark wie ein Bär alb. si arushë (wörtl. *wie eine Bärin) 'sehr starke Person' für positive Wertungen. „Die Bärin“ steht nur im sonst eher patriarchalisch geprägten albanischen Sprachraum als Symbol für Stärke. Es heisst auch nur Arusha e Madhe ,Großer Bär' (wörtl. *Große Bärin). Mehrheitlich existieren aber z. B. auch nur phraseologische Verbindungen mit dhelpër ,*Füchsin' (z.B. është dhelpër $e$ vjetër (wörtl. eine alte Füchsin sein)), nicht aber mit ,Fuchs'9. Die Tierbezeichnung in einem konkreten Phraseologismus hängt, wie diese Beispiele belegen, somit nicht nur von den Vorstellungen und Werten ab, die einem Tier kulturspezifisch in einer Sprachgemeinschaft zugeschrieben werden, sondern auch von den in einer konkreten Sprache bereits existierenden bzw. gegebenen Strukturen und pragmatischen Regelungen.

Gegenstand dieses Beitrags war auch die Feststellung zwischensprachlicher Äquivalenzbeziehungen zwischen deutschen und albanischen Phraseologismen mit Tierbezeichnungen. In der folgenden Übersicht werden die Ergebnisse der quantitativen Auswertung für die deutsch-albanischen Äquivalenzbeziehungen tabellarisch dargestellt:

\footnotetext{
${ }^{9}$ Dhelpër steht beispielsweise als Gattungsname, arushë bevorzugt in Wortverbindungen.
} 


\begin{tabular}{|l|c|c|}
\hline \multicolumn{1}{|c|}{ Äquivalenztyp } & $\begin{array}{c}\text { Anzahl der } \\
\text { Belege }\end{array}$ & $\%$ \\
\hline Volläquivalenz & 33 & 12 \\
\hline Teiläquivalenz: & & \\
- Gruppe a) & 23 & $\mathbf{8}$ \\
- Gruppe b) & 67 & $\mathbf{2 4}$ \\
- Gruppe c) & 25 & 9 \\
- Gruppe d) & 4 & 1 \\
- Gruppe e) & 3 & 1 \\
\hline Nulläquivalenz & $\mathbf{1 2 8}$ & $\mathbf{4 5}$ \\
\hline $\mathbf{2}$ & $\underline{\mathbf{2 8 3}}$ & $\underline{\mathbf{1 0 0}}$ \\
\hline
\end{tabular}

Tabelle 1: Ergebnisse der quantitativen Auswertung für die dt.-alb. Äquivalenzbeziehungen

Geht man von den drei Äquivalenztypen aus, so stellt die Nulläquivalenz den größten Anteil im Korpus (45\%). 43 \% aller deutschen Phraseologismen mit Tierbezeichnungen verfügen im Albanischen über Teiläquivalente, während $12 \%$ auf den Idealfall des Volläquivalents entfallen.

\section{Literatur}

\section{Als Quelle benutzte Wörterbücher}

Doku, Skender (1998): Fjalor frazeologjik gjermanisht-shqip. Tiranë: Botimet Enciklopedike.

Dudenredaktion (ed.) (1996): Deutsches Universalwörterbuch. Mannheim etc.: Bibliographisches Institut GmbH.

Dudenredaktion (ed.) (1998): Redewendungen und sprichwörtliche Redensarten. Mannheim etc: Bibliographisches Institut GmbH. (= Duden Band 11).

Kostallari, Androkli et al. (2002): Fjalor i gjuhës së sotme shqipe. Tiranë: ASHASH.

Mrazović, Pavica/Primorac, Ružica (1991): Deutsch-serbokroatisches phraseologisches Wörterbuch. Beograd: Narodna knjiga.

Schemann, Hans (1993): Deutsche Idiomatik. Die deutschen Redewendungen im Kontext. Stuttgart etc.: Klett.

Thomai, Jani (1999): Fjalor frazeologjik i gjuhës shqipe. Tiranë: ASHSH.

\section{Sekundärliteratur}

Burger, Harald (2010): Phraseologie. Eine Einführung am Beispiel des Deutschen. Berlin: Erich Schmidt Verlag.

Burger, Harald/Buhofer, Annelies/Sialm, Ambros (1982): Handbuch der Phraseologie. Berlin, New York: de Gruyter.

Dobrovol'skij, Dmitrij/Piirainen, Elisabeth (1997): Symbole in Sprache und Kultur: Studien zur Phraseologie aus kultursemiotischer Perspektive. Bochum: Brockmeyer.

Fleischer, Wolfgang $\left({ }^{2} 1997\right)$ : Phraseologie der deutschen Gegenwartssprache. Tübingen: Niemeyer . 
Földes, Csaba (1996): Deutsche Phraseologie kontrastiv: Intra- und interlinguale Zugänge. Heidelberg: Julius Groos Verlag. (= Deutsch im Kontrast; Bd. 15).

Földes, Csaba (2007): "Phraseme mit spezifischer Struktur". In: Burger, Harald/Dobrovol'skij, Dmitrij/Kühn, Peter/Norrick, Neal R. (eds.): Phraseologie: Ein internationales Handbuch der zeitgenössischen Forschung/Phraseology. An International Handbook of Contemporary Research. Berlin, New York: de Gruyter: 424-435. (=Handbücher zur Sprach- und Kommunikationswissenschaft/Handbooks of Linguistics and Communication Science 28.1-28.2).

Korhonen, Jarmo (2007): "Probleme der kontrastiven Phraseologie". In: Burger, Harald/Dobrovol'skij, Dmitrij/Kühn, Peter/Norrick, Neal R. (eds.): Phraseologie: Ein internationales Handbuch der zeitgenössischen Forschung/Phraseology. An International Handbook of Contemporary Research. Berlin, New York: de Gruyter: 574-589. (=Handbücher zur Sprach- und Kommunikationswissenschaft/Handbooks of Linguistics and Communication Science 28.1-28.2).

Wotjak, Barbara (1992): Verbale Phraseolexeme in System und Text. Tübingen: Niemeyer. 